\title{
Caracterización de los niños con osteosarcoma no metastásico quienes recibieron tratamiento con mifamurtida en dos instituciones de Bogotá (Colombia) entre 2014 y 2017
}

\section{Characterization of Children with Non-Metastatic Osteosarcoma Who Received Treatment with Mifamurtide in Two Institutions in Colombia between 2014 and 2017}

\author{
Leila Martínez Beltrán ${ }^{\mathrm{a}}$ \\ Médica oncóloga pediatra, Clinica Infantil Colsubsidio- \\ Hospital Militar Central, Bogotá, Colombia \\ Daniel Ozaeta Eidelman \\ Médico hematoncólogo pediatra, Hospital \\ Federico Lleras Acosta, Ibagué, Colombia \\ Natalia Lucía González SuÁrez \\ Médica oncóloga pediatra, Hospital Militar Central, \\ Bogotá, Colombia
}

a Correspondencia: leilamartinezoncologa@gmail.com

Cómo citar: Martínez Beltrán L, Ozaeta Eidelman D, González Suárez NL. Caracterización de los niños con osteosarcoma no metastásico quienes recibieron tratamiento con mifamurtida en dos instituciones de Bogotá (Colombia) entre 2014 y 2017. Univ. Med. 2019;60(4). https:// doi.org/10.11144/Javeriana.umed60-4.oste

\section{RESUMEN}

Introducción: El osteosarcoma es el tumor óseo más frecuente en los niños. La supervivencia de los que no tienen metástasis al inicio del tratamiento no ha cambiado significativamente en la última década. Existen estudios que sugieren el beneficio del uso de nuevas moléculas como mifamurtida. Métodos: Se describieron las variables de interés en 8 pacientes menores de 18 años con osteosarcoma de alto grado no metastásico, que recibieron quimioterapia convencional y mifamurtida como adyuvante en 2 instituciones de Colombia entre 2014 y 2017. Resultados: La mayoría de los pacientes tenía afectación del fémur por osteosarcoma convencional. Todos se manejaron con quimioterapia pre y posquirúrgica. El $75 \%$ de los pacientes fue llevado a salvamento de extremidad. En total se evaluaron 375 ciclos de mifamurtida a dosis de 2 $\mathrm{mg} / \mathrm{m}^{2}$ de superficie corporal total. Se presentaron efectos adversos en $7 \mathrm{de}$ los 375 ciclos administrados $(1,87 \%)$, en 4 de los 8 pacientes participantes en el estudio. Al finalizar el estudio, 6 de los 8 pacientes estaban vivos. Conclusiones: En los pacientes evaluados, el uso de mifamurtida fue bien tolerado; sin embargo, por el tipo de estudio, no se puede determinar si el uso de este medicamento tuvo impacto en la supervivencia.

Palabras clave

osteosarcoma; mifamurtida; niño; supervivencia.

\section{ABSTRACT}

Introduction: Osteosarcoma is the most frequent bone tumor in children. Survival of patients who do not have metastases at the beginning has not 
changed in the last decade; there are studies that suggest the benefit of the use of new molecules such as mifamurtide. Methods: We described the variables of interest in 8 patients under 18 years of age with high-grade non-metastatic osteosarcoma, who received management with conventional chemotherapy and mifamurtide as adjuvant in 2 Colombian institutions between 2014 and 2017. Results: The majority of the patients had femoral compromise due to conventional osteosarcoma, all of them received management with pre and post-surgical chemotherapy, $75 \%$ of the patients were taken to limb salvage. In total, 375 cycles of mifamurtide were evaluated ( $2 \mathrm{mg} / \mathrm{m}^{2}$ of total body surface area). There were adverse effects in 7 of the 375 cycles administered (1.87\%); these occurred in 4 of the 8 patients participating in the study; at the end of the study, 6 of 8 patients were alive. Conclusions: In the patients evaluated, the use of mifamurtide was well tolerated, however due to the type of study it can't be determined if the use of this medication had an impact on survival.

Keywords

osteosarcoma; mifamurtide; child; survival.

\section{Introducción}

Los tumores óseos representan aproximadamente el 6\% de las enfermedades oncológicas en las personas menores de 20 años de edad. El osteosarcoma es el tumor óseo maligno más frecuente en niños y adolescentes, y corresponde al $56 \%$ de todas las neoplasias óseas en esta población. La incidencia anual varía entre 3,5 casos por millón en niños menores de 15 años y 8,8 casos por millón en personas entre 15 y 19 años, con un pico de aparición alrededor de los 15 años de edad $(1,2)$. En Colombia, el registro poblacional de Cali reportó una incidencia ajustada por edad y sexo de 4,2 casos por millón en menores de 15 años de edad (3).

En el momento del diagnóstico, del 70\% al $80 \%$ de los pacientes presentaron enfermedad localizada, y del 20\% al 30\% tenían metástasis, entre ellas las más frecuentes: pulmonares, linfáticas y óseas (4).

El tratamiento actual del osteosarcoma de alto grado metastásico y no metastásico incluye quimioterapia neoadyuvante, seguida por resección quirúrgica completa del tumor primario, idealmente con bordes negativos y quimioterapia adyuvante. $\mathrm{Ha}$ generado controversia la elección del régimen de quimioterapia y el momento óptimo (es decir, preoperatorio-neoadyuvante frente a postoperatorio-adyuvante), pues no existe un beneficio definitivo de supervivencia para la quimioterapia neoadyuvante en comparación con la adyuvante (5); sin embargo, muchos centros utilizan preferentemente quimioterapia preoperatoria, particularmente si se está contemplando un procedimiento de preservación de la extremidad.

El grado de necrosis en la anatomía patológica refleja la efectividad de la quimioterapia neoadyuvante (4). La respuesta a la quimioterapia neoadyuvante es un factor pronóstico importante, pero no hay pruebas de que los resultados en pacientes con respuesta histológica deficiente a la quimioterapia neoadyuvante mejoren cuando se altera el régimen de quimioterapia postoperatoria (6). La capacidad de respuesta de un osteosarcoma a la quimioterapia neoadyuvante es un determinante importante del resultado clínico para la mayoría de los subtipos histológicos $(7,8,9,10,11,12,13,14,15)$.

Las tasas de supervivencia a cinco años para los pacientes con un sarcoma de extremidad y una "buena" respuesta a la quimioterapia (según lo define el $95 \%$ o más de necrosis en la pieza quirúrgica) son significativamente más altas que en aquellos con una respuesta menor (71\% a $80 \%$ contra $45 \%$ a $60 \%$, respectivamente) $(11,13,15,16)$. Sin embargo, la demostración de una relación inversa entre el valor predictivo de la necrosis tumoral y la intensidad de la terapia de inducción en un informe ha llevado a cuestionar el verdadero valor de la respuesta histológica como marcador pronóstico (17).

El objetivo terapéutico es eliminar los tumores detectables clínicamente y controlar las metástasis microscópicas con poliquimioterapia, para mejorar la supervivencia y prevenir la recurrencia. Se supuso y se demostró posteriormente que la enfermedad metastásica subclínica está presente en el momento del diagnóstico en la mayoría de los pacientes y que la quimioterapia puede erradicar con éxito estos depósitos si se inicia cuando es baja la carga de la enfermedad (18). 
Los medicamentos utilizados con más frecuencia son: cisplatino, doxorrubicina, metotrexato con rescates de leucovorina, ifosfamida y etopósido $(19,20)$. No se ha establecido el régimen óptimo; sin embargo, la evidencia disponible apoya el beneficio de tres fármacos en comparación con un régimen de dos fármacos, en particular para niños y adultos jóvenes (21).

Para niños y adolescentes, recomendamos el régimen de metotrexato más doxorrubicina y cisplatino (MAP), que se usó en el brazo de control del protocolo del Grupo de Estudio de Osteosarcoma Americano (AOST) 0331 (EURAMOS-1) (22).

El pronóstico, la mortalidad y la tasa de progresión se relacionan principalmente con la presencia de metástasis (23). La supervivencia libre de evento a los 5 años no se ha modificado significativamente en los últimos 20 años (24). Con el tratamiento actual, oscila entre el 60\% y el $70 \%$ para los pacientes con osteosarcoma no metastásico y entre el $20 \%$ y el 30\% para los que presentan osteosarcoma metastásico (4). Por lo tanto, se requieren nuevas estrategias terapéuticas para mejorar la supervivencia de estos pacientes. En ese escenario, se contempló una modificación del régimen postoperatorio en los pacientes que no lograban adecuados índices de necrosis y el pionero fue el protocolo T10 en el Memorial Sloan-Kettering Cancer Center $(25,26)$.

Sin embargo, esta consideración dejó de usarse, porque no se mantuvieron los beneficios de la estrategia T10 (27); los principales grupos cooperativos no han podido confirmar que estos cambios mejoren los resultados $(17,28,29,30,31)$, y el ensayo internacional multigrupo EURAMOS-1 no mostró beneficio de cambios de esquemas en los pacientes con deficientes respuestas histológicas (6).

En este contexto, otras consideraciones han sido estudiar fármacos inmunomoduladores con acción antitumoral, especialmente en neoplasias resistentes a la quimioterapia convencional $(19,24)$. La mifamurtida (muramil tripéptido: MPT) es un análogo sintético lipofílico del muramil dipéptido, un componente de las paredes celulares de las bacterias, con propiedades inmunoestimulantes; este fármaco incrementa la producción de citocinas proinflamatorias. Al ser lipofílico, se incorpora en la membrana de los macrófagos y monocitos, los cuales se activan específicamente contra las células tumorales, sin afectar las células normales. La MTP contribuye a la erradicación de las micrometástasis residuales que existen en los pacientes con osteosarcoma que son resistentes a la quimioterapia $(4,24,32)$.

La dosis recomendada de MTP es $2 \mathrm{mg} /$ $\mathrm{m}^{2}$ de superficie corporal, administrado por vía endovenosa en $1 \mathrm{~h}, 2$ veces a la semana durante 12 semanas; posteriormente, una vez a la semana durante 24 semanas, para un total de 36 semanas y 48 dosis (4).

Existe interés de seguir estudiando la eficacia de este agente como terapia estándar en el osteosarcoma $(33,34,35)$. Hay estudios que sugieren que la adición de MTP mejora la supervivencia libre de evento en los pacientes con osteosarcoma no metastásico $(23,33)$. No existen estudios en países de ingreso medio alto, medio bajo o bajo con este medicamento en niños con osteosarcoma.

En relación con los efectos adversos y posible toxicidad, se han reportado efectos hematológicos, hepáticos, renales, digestivos, cardiacos, del sistema nervioso, pérdida auditiva, fiebre e infección (4).

La MTP está disponible en Colombia desde 2014, y desde 2016 la Clínica Infantil Colsubsidio y el Hospital Militar Central, en Bogotá, la incluyeron en el protocolo de manejo para niños con osteosarcoma no metastásico. En este artículo se caracterizan los pacientes con osteosarcoma no metastásico que recibieron MTP como parte del tratamiento oncológico en estas dos instituciones.

\section{Materiales y métodos}

Se presenta una serie de casos (estudio observacional, descriptivo y retrospectivo) con todos los pacientes de 2 a 18 años con osteosarcoma de alto grado, no metastásico, 
que fueron llevados a resección quirúrgica macroscópicamente completa e iniciaron tratamiento con MTP adicional al protocolo convencional, entre el $1^{\text {o }}$ de enero de 2014 y el 31 de julio de 2017, en dos instituciones que manejan pacientes oncológicos en Bogotá (Colombia).

Los padres de los pacientes incluidos otorgaron el consentimiento informado, y los pacientes, el asentimiento correspondiente en los casos pertinentes. Previa autorización por parte del Comité de Ética e Investigación de las instituciones participantes, se revisaron las historias clínicas con la ayuda de los investigadores principales y el registro del valor de las variables con una base de datos en Excel $^{\circledR}$.

Se evaluaron características generales de los pacientes, sociodemográficas, aspectos oncológicos de importancia, histología tumoral, localización y tratamiento oncológico (que incluyó dosis acumulada de quimioterapia y tipo de control local); de igual manera, se recolectó información acerca de los efectos secundarios que se mencionan en la tabla 1.

\section{Tabla 1}

Definición de efectos secundarios

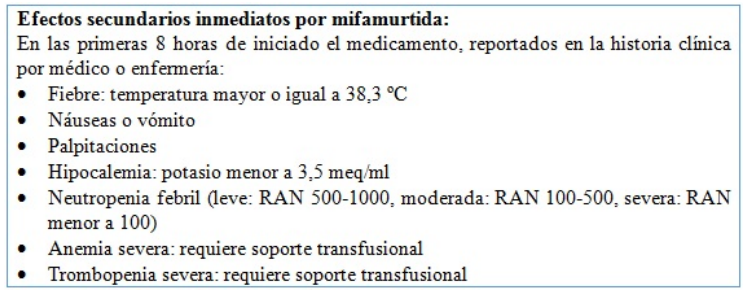

RAN:recuento absoluto de neutrófilos.

La información se recolectó y analizó utilizando Excel ${ }^{\circledR}$. Para las variables cualitativas se estimaron medidas de frecuencia y para las variables cuantitativas se calcularon medidas de tendencia central: promedio o mediana con las medidas de dispersión correspondientes.

\section{Resultados}

Se evaluaron 8 pacientes que corresponden a la totalidad de niños con diagnóstico de osteosarcoma no metastásico que ingresaron a las instituciones participantes. Los resultados se presentan en las tablas 2 y 3 .

Tabla 2

Características de los pacientes

\begin{tabular}{|c|c|c|}
\hline & Variable & n (\%) \\
\hline \multirow[t]{2}{*}{ Sexo } & Masculino & $5(62)$ \\
\hline & Femenino & $3(38)$ \\
\hline \multirow[t]{2}{*}{ Tipo histológico } & Convencional & $7(88)$ \\
\hline & Telangiectásico & $1(12)$ \\
\hline \multirow{3}{*}{ Tumor primario } & Fémur & $5(62)$ \\
\hline & Tibia & $2(25)$ \\
\hline & Húmero & $1(12)$ \\
\hline \multirow[t]{2}{*}{ Cirugía } & Salvamento & $6(75)$ \\
\hline & Amputación & $2(25)$ \\
\hline \multirow[t]{2}{*}{ Necrosis tumoral } & Mayor o igual a $90 \%$ & $2(25)$ \\
\hline & Menor a $90 \%$ & $6(75)$ \\
\hline \multirow[t]{2}{*}{ Seguimiento } & Vivos & $6(75)$ \\
\hline & Fallecidos & $2(25)$ \\
\hline Esquema mifamurtide & 48 ciclos & $7(88)$ \\
\hline $2 \mathrm{~g} / \mathrm{m}^{2}$ ciclo & 39 ciclos & $1(12)$ \\
\hline
\end{tabular}

Tabla 3

Promedio de dosis acumulada de los

quimioterapéuticos

\begin{tabular}{ll}
\hline Medicamento & Dosis acumulada \\
\hline Quimioterapia neoadyuvante & \\
\hline Doxombicina & $215 \mathrm{mg} / \mathrm{m}^{2}\left(150-375 \mathrm{mg} / \mathrm{m}^{2}\right)$ \\
\hline Cisplatino & $250 \mathrm{mg} / \mathrm{m}^{2}\left(200-360 \mathrm{mg} / \mathrm{m}^{2}\right)$ \\
\hline Ifosfamida & $16 \mathrm{~g} / \mathrm{m}^{2}\left(24-48 \mathrm{mg} / \mathrm{m}^{2}\right)$ \\
\hline Quimioterapia adyuvante & Promedio de dosis acumulada \\
\hline Doxorrubicina & $198,7 \mathrm{mg} / \mathrm{m}^{2}\left(75-300 \mathrm{mg} / \mathrm{m}^{2}\right)$ \\
\hline Cisplatino & $262,5 \mathrm{mg} / \mathrm{m}^{2}\left(240-360 \mathrm{mg} / \mathrm{m}^{2}\right)$ \\
\hline Ifosfamida & $46,8 \mathrm{~g} / \mathrm{m}^{2}\left(18-75 \mathrm{mg} / \mathrm{m}^{2}\right)$ \\
\hline Metotrexate & $42 \mathrm{~g} / \mathrm{m}^{2}\left(24-48 \mathrm{mg} / \mathrm{m}^{2}\right)$ \\
\hline Etopósido & $1500 \mathrm{mg} / \mathrm{m}^{2}\left(500-2000 \mathrm{mg} / \mathrm{m}^{2}\right)$ \\
\hline
\end{tabular}

La edad promedio de los pacientes en el momento del diagnóstico fue de 12,5 años (con intervalo de 9 a 16 años), la mayoría eran hombres y la localización más frecuente del tumor primario fue el fémur distal. El tipo histológico más común fue osteosarcoma central, subtipo convencional (de acuerdo con la clasificación de Organización Mundial de la Salud).

Todos recibieron quimioterapia neoadyuvante previa al control local. Se consideró buena respuesta cuando el porcentaje de necrosis alcanzado fue mayor al 95\%, según la clasificación Huvos. Posteriormente, se administró quimioterapia adyuvante.

En relación con la quimioterapia neoadyuvante, el esquema recibido fue doxorrubicina y cisplatino, y a 6 se les administró ifosfamida y metotrexato, según el estadio clínico, la adherencia del paciente y el protocolo institucional. Solo 2 pacientes requirieron 
amputación; los demás fueron llevados a cirugía de salvamento. En cuanto a la quimioterapia adyuvante, todos recibieron doxorrubicina; 7 , ifosfamida; 7, cisplatino; 6, metotrexato, y 5, etopósido. Todos recibieron MTP $2 \mathrm{mg} / \mathrm{m}^{2}$ por ciclo, 7 completaron 48 ciclos y uno recibió 39 ciclos.

Al finalizar el seguimiento, en agosto de 2018, 2 pacientes habían fallecido por progresión de la enfermedad con afectación metastásica pulmonar; ambos tuvieron necrosis menor al $95 \%$ en el momento del control local y uno de ellos fue el que recibió 39 ciclos de mifamurtida.

Hubo efectos adversos en 7 de los 375 ciclos administrados (1,87\%); estos ocurrieron en 4 de los 8 pacientes participantes en el estudio. Tres pacientes presentaron fiebre, anemia, trombopenia y neutropenia en las primeras 6 horas de aplicación en uno de los ciclos; un paciente, fiebre no asociada a alteraciones en el hemograma; un paciente, fiebre asociada a anemia leve, y un paciente, cefalea. Por último, un paciente reportó palpitaciones.

\section{Discusión y conclusiones}

Aunque la incidencia del osteosarcoma es baja, es el tumor óseo maligno más frecuente en los niños. Muchos casos se diagnostican de forma tardía, lo cual influye de manera importante en el pronóstico.

El esquema de tratamiento sigue siendo control local quirúrgico con quimioterapia previa y posterior al procedimiento, con medicamentos convencionales como alquilantes, platinos, antraciclinas, podofilina semisintética y análogos del ácido fólico.

A pesar de los avances científicos y los ajustes que se han realizado a los protocolos de tratamiento de la enfermedad, la supervivencia no ha cambiado significativamente en las últimas décadas, lo que hace necesario considerar estrategias terapéuticas con diferentes mecanismos de acción que influyan en el pronóstico de los niños. La MTP es un fármaco con propiedades inmunomoduladores que le confieren actividad tumoricida y existen estudios que sugieren su efectividad en pacientes con osteosarcoma.

Meyers et al. (33) publicaron en 2008 un experimento clínico para determinar si la adición de ifosfamida y MTP aumentaba la supervivencia global y la supervivencia libre de evento en pacientes con osteosarcoma no metastásico, resecable. Realizaron un diseño factorial $2 \times$ 2 , incluyendo a 662 pacientes. Compararon la quimioterapia con cisplatino, doxorrubicina y metotrexato con y sin adición de ifosfamida y MTP. La adición de MTP mejoró la supervivencia a 6 años del 70\% al 78\% (p=0,03). La razón de riesgo fue 0,71 (IC 95\%: 0,52-0,96). No encontraron diferencias estadísticamente significativas entre el esquema con ifosfamida y sin esta. Concluyeron que la adición de MTP mejoraba la sobrevida global.

En 2017, Jimmy et al. (4) publicaron una revisión sistemática de la literatura para estimar el efecto de la administración de MTP, en adición a la quimioterapia adyuvante, en la supervivencia libre de evento, supervivencia global, recurrencia y calidad de vida en pacientes con osteosarcoma de alto grado metastásico y no metastásico. Encontraron dos estudios experimentales que incluyeron 802 pacientes de todas las edades. No encontraron diferencias en la supervivencia libre de evento en osteosarcoma metastásico y no metastásico. Hubo pequeñas diferencias, pero estadísticamente significativas a favor de la MTP en la supervivencia global en pacientes con osteosarcoma no metastásico y supervivencia libre de progresión en pacientes con metástasis pulmonares o recaída. Los autores concluyeron que la evidencia para estimar la efectividad de la MTP es limitada.

El estudio de 2015 de Song et al. (23) empleó un modelo de Markov para estimar la efectividad de la adición de MTP a la quimioterapia en pacientes con osteosarcoma metastásico y no metastásico, tomando como horizonte temporal toda la vida, iniciando a los 13 años. El estudio concluyó que la adición de MTP aumenta la efectividad del tratamiento en osteosarcoma metastásico y no metastásico. La efectividad relativa fue mayor en el metastásico. 
Los estudios publicados incluyen población de diferentes edades, sin considerar a los niños como subgrupo. No existen estudios de la efectividad y los efectos secundarios de la MTP en países de ingreso medio alto o inferior. En nuestro estudio se administraron 375 ciclos a 8 pacientes con osteosarcoma no metastásico. Dos de estos pacientes fallecieron y solo en el 1,87\% de los ciclos se evidenciaron efectos secundarios; no se presentaron muertes asociadas a toxicidad por el medicamento. Es importante aclarar que se tomaron en cuenta los eventos reportados por el paciente, los médicos y el personal de enfermería, sin hacer búsqueda activa de otros eventos documentados en la literatura sobre el tema.

Considerando que es un estudio descriptivo, sus resultados tienen por objetivo la generación de hipótesis. Sin embargo, de acuerdo con la baja prevalencia de la enfermedad y con que no hay estudios en países en vías de desarrollo, es valioso para la comunidad científica informar la experiencia con los niños tratados con MTP en las instituciones mencionadas. En Colombia, aunque existen algunas otras instituciones, además de las mencionadas en el estudio, que han utilizado la MTP, no hay ningún estudio publicado al respecto.

\section{Declaración de conflicto de interés}

La Compañía Farmacéutica Takeda apoyó el proceso de publicación de este artículo.

\section{Agradecimientos}

El manejo de los pacientes se realizó según el criterio clínico de los médicos tratantes, por lo cual agradecemos a la Clínica Infantil Colsubsidio y al Hospital Militar Central.

\section{Referencias}

1. Lanzkowsky P, Lipton JM, Fish JD. Manual of pediatric hematology and oncology. 6th ed. s. 1.: Academic Press; 2016.

2. Pizzo PA, Poplack DG. Principles and practice of pediatric oncology. 7th ed. Philadelphia: Lippincott Williams and Wilkins; 2015.

3. Suárez A, Soto C, Gómez L, Gamboa Ó, Soto D, Escandón S, et al. Resultados del tratamiento de osteosarcoma convencional de alto grado en niños y adolescentes: análisis de supervivencia de una cohorte tratada sin metotrexato. Rev Colomb Cancerol. 2017;21(2):86-94.

4. Jimmy R, Stern C, Lisy K, White $\mathrm{S}$. Effectiveness of mifamurtide in addition to standard chemotherapy for high-grade osteosarcoma. JBI Database Syst Rev Implement Reports. 2017 Aug;15(8):2113-52.

5. Goorin AM, Schwartzentruber DJ, Devidas M, Gebhardt MC, Ayala AG, Harris MB, et al. Presurgical chemotherapy compared with immediate surgery and adjuvant chemotherapy for nonmetastatic osteosarcoma: Pediatric Oncology Group Study POG-8651. J Clin Oncol. 2003;21(8):1574-80.

6. Marina NM, Smeland S, Bielack SS, Bernstein M, Jovic G, Krailo $\mathrm{MD}$, et al. Comparison of MAPIE versus MAP in patients with a poor response to preoperative chemotherapy for newly diagnosed high-grade osteosarcoma (EURAMOS-1): An open-label, international, randomised controlled trial. Lancet Oncol. 2016 Oct;17(10):1396-408.

7. Rosen G. Preoperative (neoadjuvant) chemotherapy for osteogenic sarcoma: A ten year experience. Orthopedics. 1985;8(5):659-64.
8. Bielack SS, Kempf-Bielack
B, Winkler K. Osteosarcoma: 
Relationship of response to preoperative chemotherapy and type of surgery to local recurrence. J Clin Oncol. 1996;14(2):683-4.

9. Picci P, Sangiorgi L, Rougraff BT, Neff JR, Casadei R, Campanacci M. Relationship of chemotherapy-induced necrosis and surgical margins to local recurrence in osteosarcoma. J Clin Oncol. 1994;12(12):2699-705.

10. Kawai A, Healey JH, Boland PJ, Lin PP, Huvos AG, Meyers PA. Prognostic factors for patients with sarcomas of the pelvic bones. Cancer. 1998;82(5):851-9.

11. Bacci G, Bertoni F, Longhi A, Ferrari S, Forni C, Biagini R, et al. Neoadjuvant chemotherapy for high-grade central osteosarcoma of the extremity. Cancer. 2003;97(12):3068-75.

12. Bacci G, Picci P, Ruggieri P, Mercuri M, Avella M, Capanna $\mathrm{R}$, et al. Primary chemotherapy and delayed surgery (neoadjuvant chemotherapy) for osteosarcoma of the extremities. The Istituto Rizzoli Experience in 127 patients treated preoperatively with intravenous methotrexate (high versus moderate doses) and intraarterial cisplatin. Cancer. 1990;65(11):2539-53.

13. Bielack SS, Kempf-Bielack B, Delling G, Exner GU, Flege S, Helmke $\mathrm{K}$, et al. Prognostic factors in highgrade osteosarcoma of the extremities or trunk: An analysis of 1,702 patients treated on neoadjuvant cooperative osteosarcoma study group protocols. J Clin Oncol. 2002;20(3):776-90.

14. Petrilli AS, de Camargo B, Filho VO, Bruniera P, Brunetto AL, Jesus-Garcia R, et al. Results of the Brazilian Osteosarcoma Treatment Group Studies III and IV: Prognostic factors and impact on survival. J Clin Oncol. 2006;24(7):1161-8.
15. Hauben EI, Weeden S, Pringle J, Van Marck EA, Hogendoorn PC. Does the histological subtype of high-grade central osteosarcoma influence the response to treatment with chemotherapy and does it affect overall survival? Eur J Cancer. 2002;38(9):1218-25.

16. Bacci G, Longhi A, Versari M, Mercuri M, Briccoli A, Picci P. Prognostic factors for osteosarcoma of the extremity treated with neoadjuvant chemotherapy. Cancer. 2006;106(5):1154-61.

17. Bishop MW, Chang Y-C, Krailo MD, Meyers PA, Provisor AJ, Schwartz $\mathrm{CL}$, et al. Assessing the prognostic significance of histologic response in osteosarcoma: A comparison of outcomes on CCG-782 and INT0133. A report from the Children's Oncology Group Bone Tumor Committee. Pediatr Blood Cancer. 2016;63 (10):1737-43.

18. Bruland OS, Høifødt $\mathrm{H}$, Saeter G, Smeland S, Fodstad O. Hematogenous micrometastases in osteosarcoma patients. Clin Cancer Res. 2005;11(13):4666-73.

19. Frampton JE. Mifamurtide: A review of its use in the treatment of osteosarcoma. Pediatr Drugs. 2010 Jun;12(3):141-53.

20. Meyers PA. Muramyl tripeptide (mifamurtide) for the treatment of osteosarcoma. Expert Rev Anticancer Ther. 2009 Aug 10;9(8):1035-49.

21. Anninga JK, Gelderblom $\mathrm{H}$, Fiocco M, Kroep JR, Taminiau AHM, Hogendoorn PCW, et al. Chemotherapeutic adjuvant treatment for osteosarcoma: Where do we stand? Eur J Cancer. 2011;47 (16):2431-45.

22. Clinical Trials. Combination chemotherapy, PEG-interferon alfa $2 \mathrm{~b}$, and surgery in treating patients 
with osteosarcoma [internet]. 2015 [Citado 2011 jul 19]. Disponible en: http://www.clinicaltrials.gov/ct2/sh ow/NCT00134030?term $=$ AOST +03 31 \&rank $=1$

23. Song HJ, Lee JA, Han E, Lee E-K. Lifetime effectiveness of mifamurtide addition to chemotherapy in nonmetastatic and metastatic osteosarcoma: a Markov process model analysis. Tumor Biol. 2015 Sep 3;36(9):6773-9.

24. Ando K, Mori K, Corradini N, Redini F, Heymann D. Mifamurtide for the treatment of nonmetastatic osteosarcoma. Expert Opin Pharmacother. 2011 Feb 13;12(2):285-92.

25. Rosen G, Marcove RC, Huvos AG, Caparros BI, Lane JM, Nirenberg A, et al. Primary osteogenic sarcoma: Eight-year experience with adjuvant chemotherapy. J Cancer Res Clin Oncol. 1983;106(S1):55-67.

26. Rosen G, Caparros B, Huvos AG, Kosloff C, Nirenberg A, Cacavio A, et al. Preoperative chemotherapy for osteogenic sarcoma: selection of postoperative adjuvant chemotherapy based on the response of the primary tumor to preoperative chemotherapy. Cancer. 1982;49(6):1221-30.

27. Meyers PA, Heller G, Healey J, Huvos A, Lane J, Marcove R, et al. Chemotherapy for nonmetastatic osteogenic sarcoma: the Memorial Sloan-Kettering experience. J Clin Oncol. 1992;10(1):5-15.

28. Saeter G, Alvegård TA, Elomaa I, Stenwig AE, Holmström T, Solheim OP. Treatment of osteosarcoma of the extremities with the T-10 protocol, with emphasis on the effects of preoperative chemotherapy with single-agent high-dose methotrexate: a Scandinavian Sarcoma Group study. J Clin Oncol. 1991;9(10):1766-75.
29. Winkler K, Beron G, Delling G, Heise U, Kabisch $H$, Purfürst C, et al. Neoadjuvant chemotherapy of osteosarcoma: results of a randomized cooperative trial (COSS-82) with salvage chemotherapy based on histological tumor response. J Clin Oncol. 1988;6(2):329-37.

30. Souhami RL, Craft AW, Van der Eijken JW, Nooij M, Spooner D, Bramwell VH, et al. Randomised trial of two regimens of chemotherapy in operable osteosarcoma: A study of the European Osteosarcoma Intergroup. Lancet. 1997;350(9082):911-7.

31. Smeland S, Müller C, Alvegard TA, Wiklund T, Wiebe T, Björk $\mathrm{O}$, et al. Scandinavian Sarcoma Group Osteosarcoma Study SSG VIII: Prognostic factors for outcome and the role of replacement salvage chemotherapy for poor histological responders. Eur J Cancer. 2003;39(4):488-94.

32. Anderson PM, Tomaras M, McConnell K. Mifamurtide in osteosarcoma - A practical review. Drugs of Today. 2010 May;46(5):327.

33. Meyers PA, Schwartz CL, Krailo MD, Healey JH, Bernstein ML, Betcher $\mathrm{D}$, et al. Osteosarcoma: the addition of muramyl tripeptide to chemotherapy improves overall survival--a report from the Children's Oncology Group. J Clin Oncol. 2008 Feb 1;26(4):633-8.

34. Bielack SS, Marina N, Ferrari S, Helman LJ, Smeland S, Whelan JS, et al. Osteosarcoma: The Same Old Drugs or More? J Clin Oncol. 2008;26(18):3102-3.

35. Hunsberger S, Freidlin B, Smith MA. Complexities in interpretation of osteosarcoma clinical trial results. J Clin Oncol. 2008;26(18):3103-4. 